\title{
Scoring model of convex probe endobronchial ultrasound multimodal imaging in differentiating benign and malignant lung lesions
}

\author{
Xinxin Zhi ${ }^{1,2,3 \#}$, Lei Wang ${ }^{4 \#}$, Junxiang Chen ${ }^{1,2,3}$, Xiaoxuan Zheng ${ }^{1,2,3}$, Ying Li $^{1,2,3}$, Jiayuan Sun $^{1,2,3}$ \\ ${ }^{1}$ Department of Respiratory Endoscopy, Shanghai Jiao Tong University, Shanghai, China; ${ }^{2}$ Department of Respiratory and Critical Care Medicine, \\ Shanghai Chest Hospital, Shanghai Jiao Tong University, Shanghai, China; ${ }^{3}$ Shanghai Engineering Research Center of Respiratory Endoscopy, \\ Shanghai, China; ${ }^{4}$ Department of Ultrasound, Shanghai Chest Hospital, Shanghai Jiao Tong University, Shanghai, China \\ Contributions: (I) Conception and design: J Sun, X Zhi; (II) Administrative support: J Sun; (III) Provision of study materials or patients: J Chen, Y Li, J \\ Sun; (IV) Collection and assembly of data: X Zhi; (V) Data analysis and interpretation: X Zhi, L Wang, J Chen; (VI) Manuscript writing: All authors; \\ (VII) Final approval of manuscript: All authors. \\ "These authors contributed equally to this work. \\ Correspondence to: Jiayuan Sun, PhD. Department of Respiratory Endoscopy, Shanghai Jiao Tong University, Shanghai, China; Department of \\ Respiratory and Critical Care Medicine, Shanghai Chest Hospital, Shanghai Jiao Tong University, 241 West Huaihai Road, Shanghai 200030, China. \\ Email: xkyyjysun@163.com.
}

Background: Convex probe endobronchial ultrasound images can reflect the morphology, blood flow status and stiffness of the lesions. Endobronchial ultrasound multimodal imaging has great value for the diagnosis of intrathoracic lymph nodes. This study aimed to analyze the application of endobronchial ultrasound multimodal imaging on lung lesions.

Methods: Patients undergoing endobronchial ultrasound-guided transbronchial needle aspiration in Shanghai Chest Hospital from July 2018 to December 2019 were retrospectively enrolled. Nine grayscale features (long and short axes, margin, shape, lobulation sign, echogenicity, necrosis, liquefaction, calcification, and air-bronchogram), blood flow volume and elastography five-score method were analyzed to explore the best diagnostic method. The gold standard for diagnosing lesions depends on the histological and cytopathological findings of endobronchial ultrasound-guided transbronchial needle aspiration, transthoracic biopsy, resected sample of lesions, microbiological examination or clinical follow-up of at least 6 months.

Results: Endobronchial ultrasound multimodal imaging of 97 malignant lung lesions and 19 benign lung lesions from 116 patients were analyzed. There were statistically significant differences in distinct margin, presence of lobulation sign, presence of necrosis, and elastography grading score 4-5 between malignant and benign lung lesions, among which presence of lobulation sign and elastography grading score 4-5 were independent predictors. A diagnostic scoring model was then constructed based on the above four features, and when two or more features were present, the sensitivity, specificity, positive predictive value, negative predictive value and accuracy for malignant lung lesions prediction were $92.78 \%, 57.89 \%, 91.84 \%, 61.11 \%$ and $87.07 \%$, respectively.

Conclusions: The combination of endobronchial ultrasound grayscale and elastography has potential value for malignant and benign lung lesions differentiation. The diagnostic scoring model established in this study needs further validation to guide the malignant and benign diagnosis of lung lesions.

Keywords: Endobronchial ultrasound-guided transbronchial needle aspiration (EBUS-TBNA); grayscale; elastography; lung lesions; diagnosis

Submitted Nov 27, 2020. Accepted for publication Dec 17, 2020.

doi: 10.21037/jtd-2020-abpd-005

View this article at: http://dx.doi.org/10.21037/jtd-2020-abpd-005 


\section{Introduction}

Lung cancer is the most commonly diagnosed cancer and the leading cause of cancer deaths worldwide, and the diagnosis of pulmonary lesions is an important medical problem $(1,2)$. Compared to chest computed tomography (CT) scan and radiography, ultrasonography can clearly reflect the internal structures of lung lesions in real-time and guide the biopsy (3).

Transthoracic ultrasound can be performed for peripheral lesions close to the chest wall (4). On account of lung tissue naturally containing gas, ultrasonic attenuate for lesions far away from the chest wall. Transthoracic ultrasound was not suitable for the examination of such lesions in the past. With the development of bronchoscopy, endobronchial ultrasound (EBUS) has played an important role in diagnosing pulmonary lesions $(5,6)$. The thinner radio probe EBUS (RP-EBUS) can enter the distal bronchus for pulmonary lesions examination, while convex probe EBUS (CP-EBUS) can examine the lesions adjacent to the trachea or main bronchus $(7,8)$. Study found that echoes, margin, vascular, bronchial patency, and hyperechoic areas were related to histology of lung lesions (7). Endoscopic ultrasonography has previously been widely examined in the internal structures of pancreatic lesions, suggesting the histopathological status of lesions $(9,10)$.

CP-EBUS has three modes including grayscale, blood flow and elastography (11). CP-EBUS imaging has been widely used in the diagnosis of intra-thoracic lymph nodes $(\mathrm{LNs})$, and relevant guidelines indicate that in patients undergoing EBUS-guided transbronchial needle aspiration (EBUS-TBNA), EBUS sonographic features can be used to distinguish malignant from benign diagnoses (12). Grayscale can reflect morphological characteristics, such as internal echoes, bronchi and margin, while Doppler imaging can reflect internal blood supply. Elastography can display the degree of tissues deformation in the form of colorful images, reflecting the relative stiffness of tissues. The corresponding colors from low relative stiffness to high relative stiffness are red/yellow, green and blue successively (13). Elastography is widely used in the whole body such as thyroid, breast, liver, prostate, and LNs (14). CP-EBUS elastography demonstrated a significant diagnostic value in intrathoracic LNs (15). The qualitative elastography method proposed by Sun $e t$ al. classified elastography images into five categories, which was convenient for clinical application, and the diagnostic accuracy for intrathoracic malignant and benign LNs was $83.82 \%$ (16).

Few studies about CP-EBUS multimodal imaging were conducted on intrapulmonary lesions and the diagnostic value is worthy of further studies. This study aims to analyze CP-EBUS multimodal features in the differentiation of malignant and benign lung mass and construct a scoring model to guide clinical application.

We present the following article in accordance with the STARD reporting checklist (available at http://dx.doi. org/10.21037/jtd-2020-abpd-005).

\section{Methods}

\section{Patients}

This was a retrospective observational single-center study. Consecutively enrolled pulmonary lesions performed by EBUS-TBNA with integrated grayscale, blood flow Doppler, and elastography videos at Shanghai Chest Hospital from July 2018 to December 2019. Lung lesions that had been diagnosed with malignancy or had undergone antitumor therapy before EBUS-TBNA were excluded. Mediastinal lesions and intrathoracic LNs were not included in this study. EBUS-TBNA was performed on patients meeting the following criteria: chest $\mathrm{CT}$ showing enlarged unknown reason pulmonary lesions with or without positron emission tomography (PET)/CT showing increased FDG uptake [standardized uptake value (SUV) $\geq 2.5$ ]; pathological confirmation was clinically required and EBUS-TBNA examination was feasible; Patients signed written informed consent to undergo EBUS-TBNA. Patients having contraindications to EBUS-TBNA were excluded.

The study was conducted in accordance with the Declaration of Helsinki (as revised in 2013). The study was approved by institutional ethics board of Shanghai Chest Hospital (NO.: KS1946) and individual consent for this retrospective analysis was waived.

\section{Videos acquisition and EBUS-TBNA operation}

An ultrasound probe with a scanning frequency of $10 \mathrm{MHz}$ was used and images were processed by ultrasonic host (EU-ME2, Olympus, Tokyo, Japan) for video acquisition. Biopsy of pulmonary lesions in this study was performed under the ultrasound bronchoscope (BF-UC260FW, Olympus, Tokyo, Japan). Bronchoscopist scanned lesions with grayscale mode and measured the long and short axes at the maximal cross section firstly, and then recorded a 10 -s gray-scale, a 20-s blood flow Doppler and two 20 -s elastography videos respectively. The grayscale 
video should reflect the entire LN status maximumly, and Doppler video should be in the cross-section with the most abundant blood flow. Elastography shall remain at the maximal cross-section of the target lesion. Two-thirds to threequarters of the Doppler and the elastography images shall be occupied by the lesion $(16,17)$. EBUS-TBNA was performed after the ultrasonography. Subsequently, the cytological and histological specimens after puncture were sent to the pathology department for examination. The gold standard for diagnosing lesions was dependent on the histological and cytopathological findings of EBUS-TBNA, transthoracic biopsy, resected sample of lesions, microbiological examination or clinical follow-up of at least 6 months.

\section{Analysis of videos}

The following indicators were recorded after the collection of multimodal videos based on previous studies: grayscale (17-23): (I) long and short axes (long axis is the longest diameter of the lesion, short diameter is the maximum diameter perpendicular to the long diameter); (II) margin: more than $50 \%$ of the boundary that can be well-defined on the ultrasound image belongs to distinct margin, otherwise it is indistinct; (III) shape: circular is defined as round or oval shape, and irregular means polygonal or complex shape based on chest CT definitions; (IV) lobulation sign: presence of lobulation is defined as $>3$ undulations, otherwise it is absent; (V) echogenicity: homogeneous means the consistency of internal echo except for the internal blood vessels and air-bronchogram, otherwise as heterogeneous; (VI) necrosis: present as hyperechoic, hypoechoic or anechoic areas without any Doppler acquisition within the lesions, otherwise it is absent; (VII) liquefaction: present as hypoechoic regions without blood flow within lesions; (VIII) calcification: presence of hyperechoic structure of various shapes with acoustic shadows on ultrasound image, otherwise it is absent; (IX) air-bronchogram: presence of laminar and regular hyperechoic concentric short lines on the hypoechoic background, otherwise as absent. Blood flow volume was categorized based on breast cancer (24): grade 0 , no blood flow; grade 1, punctiform blood flow; grade 2, less than four small vessels with or without punctiform blood flow; grade 3, four or more vessels were visualized within the lung lesion. Grade $0-1$ is considered as a benign indicator while $2-3$ as a malignant indicator. The qualitative elastography was based on the five-score method of intrathoracic LNs (16). All sonographic features above were evaluated based on videos.

\section{Assessment process of sonographic features}

In this study, three experts evaluated the above features of pulmonary lesions twice independently blind to other clinical data and the final diagnosis, and then intra-observer agreement was calculated. Each expert gave a final value for the two assessments' different results, which was used for calculating the inter-observer agreement among the three experts. Kappa values range from 0 to 1 based on Landis et al: : $0-0.20$, slight agreement; $0.21-0.40$, fair; $0.41-0.60$, moderate; 0.61-0.80, substantial; 0.81-1.00, perfect agreement (25). Different results among the three experts were discussed to determine the final result together used for subsequent analysis.

\section{Statistical analysis}

Chi-square test or Fisher's exact test were used for categorical variables. Elastography was counted as a dichotomous variable, among which scores 1-3 were considered as benign indicators and scores 4-5 as malignant indicators. Further binary logistic regression was performed for the categorical variables with statistical significance in univariate analysis or significant clinical value. Statistical differences of continuous variables including long and short axes were tested by independent sample $t$-test. $\mathrm{P}<0.05$ was considered statistically significant for all the above statistics. The receiver operating characteristic (ROC) curve was performed on continuous variables to analyze the optimal cutoff value and area under the curve (AUC) in identifying the benign and malignant pulmonary masses (26). ROC curve analysis was performed to further analyze the diagnostic value of the qualitative elastography method on pulmonary lesions. All continuous variables took data corresponding to the largest Yoden's index as cutoff values. Sensitivity, specificity, positive predictive value (PPV), negative predictive value (NPV) and accuracy were calculated by the corresponding formulas. In addition, Cohen's Kappa method was used for consistency analysis (27). All statistics were performed on SPSS version 25.0 (IBM Corp., Armonk, NY, USA).

\section{Results}

\section{Baseline patients and lesions characteristics}

EBUS multimodal imaging and EBUS-TBNA were performed on 116 lung lesions from 116 patients (85 males and 31 females with mean ages of $62.60 \pm 9.95$ and $62.87 \pm 11.49$ years, respectively, $\mathrm{P}=0.901)$. As shown in Table 1, 
Table 1 Baseline characteristic of lung lesions

\begin{tabular}{|c|c|}
\hline Characteristic & Value \\
\hline \multicolumn{2}{|l|}{ EBUS size (long axis, mean $\pm \mathrm{SD}, \mathrm{mm}$ ) } \\
\hline Malignant lesions & $29.30 \pm 6.58$ \\
\hline Benign lesions & $24.76 \pm 7.08$ \\
\hline \multicolumn{2}{|l|}{ EBUS size (short axis, mean \pm SD, mm) } \\
\hline Malignant lesions & $24.27 \pm 6.72$ \\
\hline Benign lesions & $18.13 \pm 5.69$ \\
\hline \multicolumn{2}{|l|}{ CT size ${ }^{*}$ (long axis, mean $\pm S D, m m$ ) } \\
\hline Malignant lesions & $50.46 \pm 18.96$ \\
\hline Benign lesions & $40.78 \pm 13.24$ \\
\hline \multicolumn{2}{|l|}{ CT size ${ }^{*}$ (short axis, mean $\pm \mathrm{SD}, \mathrm{mm}$ ) } \\
\hline Malignant lesions & $35.90 \pm 12.64$ \\
\hline Benign lesions & $24.81 \pm 7.34$ \\
\hline \multicolumn{2}{|l|}{ Location, No. (\%) } \\
\hline Left upper lobe & $12(10.34)$ \\
\hline Left lower lobe & $28(24.14)$ \\
\hline Right upper lobe & $45(38.79)$ \\
\hline Right middle lobe & $4(3.45)$ \\
\hline Right lower lobe & $27(23.28)$ \\
\hline \multicolumn{2}{|l|}{ Diseases, No. (\%) } \\
\hline Adenocarcinoma & $49(42.24)$ \\
\hline Squamous carcinoma & $19(16.38)$ \\
\hline Adenosquamous carcinoma & $1(0.86)$ \\
\hline NSCLS-NOS & $4(3.45)$ \\
\hline Small cell carcinoma & $16(13.79)$ \\
\hline $\begin{array}{l}\text { Combined large cell neuroendocrine } \\
\text { carcinoma }\end{array}$ & $1(0.86)$ \\
\hline Large cell carcinoma & $1(0.86)$ \\
\hline Unknown type of lung cancer & $3(2.59)$ \\
\hline Typical carcinoid tumor & $1(0.86)$ \\
\hline $\begin{array}{l}\text { Neuroendocrine tumor not otherwise } \\
\text { specified }\end{array}$ & $2(1.72)$ \\
\hline Inflammation & $8(6.90)$ \\
\hline Tuberculosis & $10(8.62)$ \\
\hline Aspergillosis & $1(0.86)$ \\
\hline
\end{tabular}

*, CT sizes were measures on 93 malignant lesions and 17 benign lesions. NSCLS-NOS, non-small cell lung cancer not otherwise specified; EBUS, endobronchial ultrasound; SD, standard deviation; CT, computed tomography. lung lesions in the right upper lobe accounted for the largest proportion (38.79\%). Only 19 benign lesions were analyzed in this study including 8 nonspecific lymphadenitis, 10 tuberculosis and 1 aspergillosis. Adenocarcinoma accounted for the largest proportion of malignant mass, followed by squamous cell carcinoma and small cell carcinoma.

\section{Intra- and inter-observer agreement}

All sonographic features assessed in this study had a perfect intra-observer agreement with $\mathrm{k}$ values greater than 0.81 . For inter-rater agreement, distinct margin, lobulation sign, echogenicity, necrosis, liquidation and calcification had substantial agreement. In addition, shape, air-bronchogram, blood flow volume and elastography grading score had perfect agreement (Table 2).

\section{EBUS multimodal sonographic features findings for lung lesions}

Figure 1 displays eight features of the grayscale mode, except for the measurement of long and short axes. Univariate analysis revealed significant statistical differences in margin, lobulation sign, necrosis and elastography grading score to diagnose malignant and benign lung lesions, but not in blood flow volume (Table 3). Multivariate analysis found that both lobulation sign and elastography grading score were independent predictive factors for malignant lesions (Table 3). Table 4 shows statistical differences between the long and short axes of the benign and malignant lesions. The ROC curves were performed and the best cutoff value for long and short axes were 29.20 and $25.41 \mathrm{~mm}$, respectively (Figure 2). The elastography grading score was $3.42 \pm 0.61$ for benign and $3.87 \pm 0.55$ for malignant lung lesions, among which squamous cell carcinoma, adenocarcinoma, small cell carcinoma, inflammation and tuberculosis were $3.89 \pm 0.57,3.86 \pm 0.50,3.81 \pm 0.54,3.25 \pm 0.71$ and $3.50 \pm 0.53$, respectively. Figure 3 shows the overall distribution of benign and malignant lesions in the elastography grading score method, which corresponded to the high sensitivity and low specificity of the qualitative method.

\section{EBUS scoring model construction}

To further explore whether the combination of features could achieve better diagnostic value, diagnostic performance analysis was performed on features with significant statistical differences. Results in Table 5 showed that when two or 
Table 2 Intra- and inter-observer agreement of sonographic features

\begin{tabular}{lcc}
\hline Characteristic & Intra-observer agreement & Inter-observer agreement \\
\hline Margin & 0.839 (perfect) & 0.672 (substantial) \\
Shape & 0.909 (perfect) & 0.844 (perfect) \\
Lobulation sign & 0.919 (perfect) & 0.633 (substantial) \\
Echogenicity & 0.935 (perfect) & 0.681 (substantial) \\
Necrosis & 0.907 (perfect) & 0.620 (substantial) \\
Liquidation & 0.898 (perfect) & 0.792 (substantial) \\
Calcification & 0.914 (perfect) & 0.795 (substantial) \\
Air-bronchogram & 0.940 (perfect) & 0.854 (perfect) \\
Blood flow volume & 0.985 (perfect) & 0.970 (perfect) \\
Elastography grading score & 0.951 (perfect) & 0.886 (perfect) \\
\hline
\end{tabular}
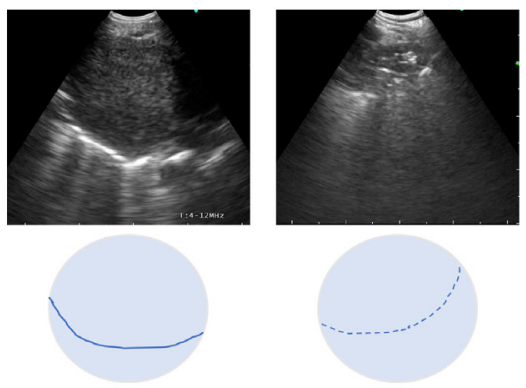

Distinct margin
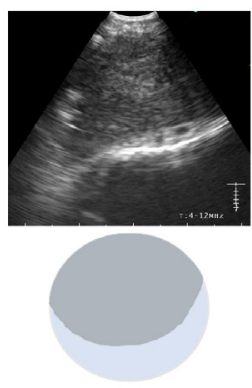

Homogeneous
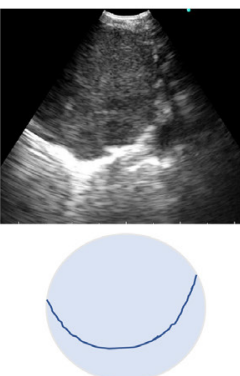

Circular shape
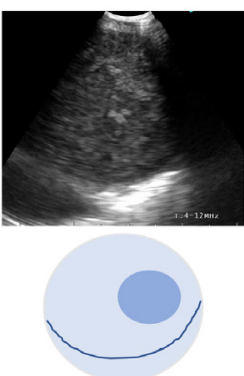

Present of necrosis
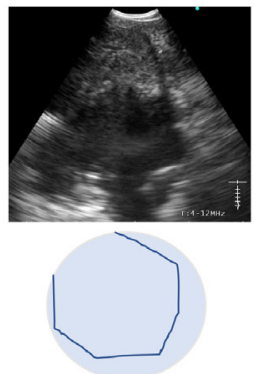

Irregular shape
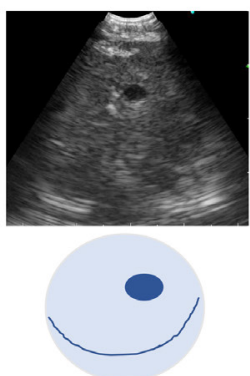

Present of liquidation
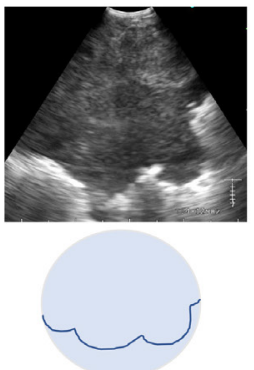

Present of lobulation sign
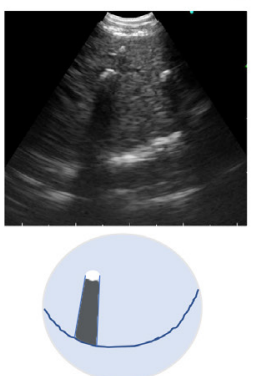

Present of calcification
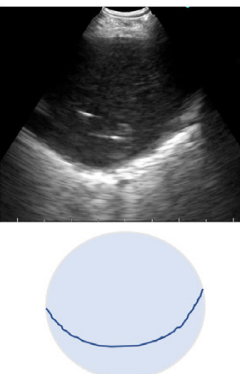

Absent of lobulation sign
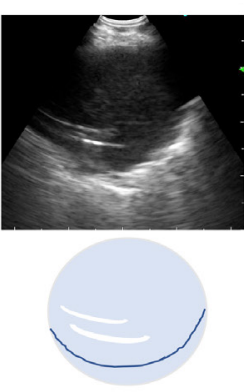

Air-bronchogram

Figure 1 Diagram of CP-EBUS grayscale features for pulmonary lesions. CP-EBUS, convex probe endobronchial ultrasound.

more of the four features including distinct margin, presence of necrosis, presence of lobulation sign and elastography grading score 4-5 appeared, the sensitivity, specificity, PPV, NPV and accuracy for malignant lesions prediction were $92.78 \%, 57.89 \%, 91.84 \%, 61.11 \%$ and $87.07 \%$, respectively.

\section{Discussion}

CP-EBUS high-frequency probe and multiple ultrasonic modes make it possible to clearly observe the boundary and internal structures, blood vessels and deformation degree of pulmonary lesions. A total of nine grayscale features, blood flow distribution and elastography grading score were explored in this study and the combination of four statistically valuable features guided the clinical diagnosis of lung lesions.

Tumor lesions usually grow expansively by squeezing the surrounding normal tissues to form a distinct boundary 
Table 3 Distribution and diagnostic value of EBUS sonographic features

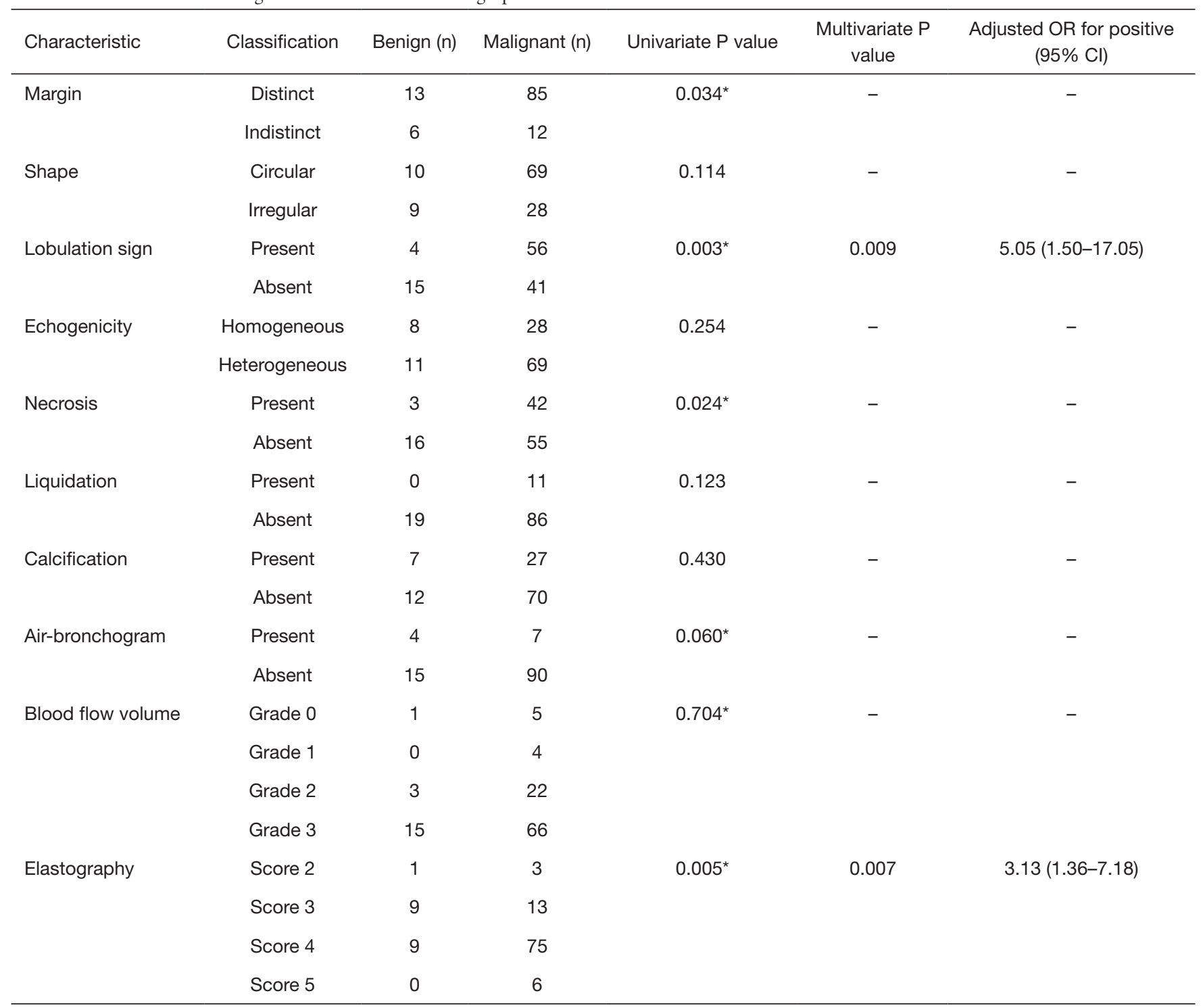

${ }^{*}$, indicators that were statistically different in univariate analysis, or that were considered clinically valuable by the bronchoscopist were then used for subsequent multivariate regression analysis. EBUS, endobronchial ultrasound; OR, odds ratio; $\mathrm{Cl}$, confidence interval.

Table 4 Analysis of the optimal diagnostic performance of continuous variables

\begin{tabular}{lcccccccc}
\hline Characteristics & P value & Cutoff & AUC & Sen (\%) & Spe (\%) & PPV (\%) & NPV (\%) & Acc (\%) \\
\hline Long axis & 0.008 & 29.20 & $0.701(0.579-0.823)$ & 61.86 & 84.21 & 95.24 & 30.19 & 65.52 \\
Short axis & 0.000 & 25.41 & $0.750(0.650-0.850)$ & 48.45 & 94.74 & 95.24 & 26.47 & 56.03 \\
Elastography & 0.005 & 3.50 & $0.692(0.556-0.827)$ & 83.51 & 52.63 & 90.00 & 38.46 & 78.45 \\
\hline
\end{tabular}

AUC, area under the curve; Sen, sensitivity; Spe, specificity; PPV, positive predictive value; NPV, negative predictive value; Acc, accuracy. 
Table 5 Construction of CP-EBUS scoring model for lung lesions

\begin{tabular}{|c|c|c|c|c|c|}
\hline Characteristics & Sen $(\%)$ & Spe (\%) & PPV (\%) & NPV (\%) & Acc $(\%)$ \\
\hline Present of necrosis & 43.30 & 84.21 & 93.33 & 22.54 & 50.00 \\
\hline Present of lobulation sign & 57.73 & 78.95 & 93.33 & 26.79 & 61.21 \\
\hline Elastography grading score $4-5$ & 83.51 & 52.63 & 90.00 & 38.46 & 78.45 \\
\hline 0 & 3.09 & 84.21 & 50.00 & 14.55 & 16.38 \\
\hline $1+$ & 96.91 & 15.79 & 85.45 & 50.00 & 83.62 \\
\hline $2+$ & 92.78 & 57.89 & 91.84 & 61.11 & 87.07 \\
\hline $3+$ & 60.82 & 78.95 & 93.65 & 28.30 & 63.79 \\
\hline
\end{tabular}

CP-EBUS, convex probe endobronchial ultrasound Sen, sensitivity; Spe, specificity; PPV, positive predictive value; NPV, negative predictive value; Acc, accuracy.

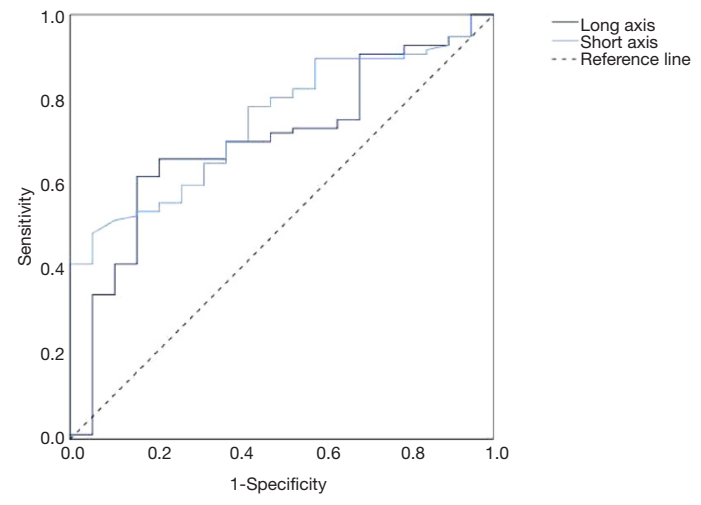

Figure 2 ROC curves analysis of long and short axes for lung lesions. The cutoff values of long and short axes were 29.20 and $25.41 \mathrm{~mm}$, respectively. ROC, receiver operating characteristic.

with a round or oval shape. Tumors will demonstrate the lobulation sign when they grow large enough (18). On the contrary, infectious tissues can disseminate through the bronchi leading to irregular shapes with indistinct boundaries $(18,19)$. In this study, well-defined margin and the presence of lobulation sign were predictive indicators of malignancy. However, due to a limited scanning angle of $60^{\circ}$, the ultrasonic image could not display the integrated shape of the targeted lesion; thus, the shape had limited diagnostic value. Moreover, the multivariate analysis showed that the lobulation sign was an independent predictor of malignancy. Necrosis and calcification in benign lesions all led to heterogeneity. Calcification often occurred in tuberculous granuloma and calcification it occurred in $50 \%$ of tuberculosis cases in this study (28). Therefore, echogenicity in this study had a limited value in predicting malignancy. Echo-free areas in RP-EBUS images corresponded to necrosis (7). Studies showed that necrosis on CP-EBUS images was a predictor of intrathoracic malignant LNs (29). Similar results were obtained in this study, and necrosis had a diagnostic value in differentiating benign from malignancy. The long and short axes were commonly analyzed in intrathoracic LNs. Studies found that the short diameter $>10 \mathrm{~mm}$ was a predictor of malignant LNs $(17,29,30)$. However, due to the natural difference in structure between lung tissue and LNs, although the long and short axes had AUC values of 0.701 and 0.750 , respectively, the clinical value was limited because the range of inflammation could generally be large. The short axis was not included into the model construction, because the size of short axis varies greatly between EBUS image and chest CT. The size on EBUS imaging cannot accurately reflect the characteristics of the pulmonary lesions, and quantitative indicators in scoring model are inconvenient for real-time application. An air-bronchogram is seen as linear hyperechoic areas in ultrasound images and it usually appears in pneumonia (7). Multiple millimetric hyperechoic structures can be created on the ultrasonographic images due to the air trapped in the small airway (19,31). Peripheral lung lesions demonstrated that the absence of linear-discrete air bronchogram was an 


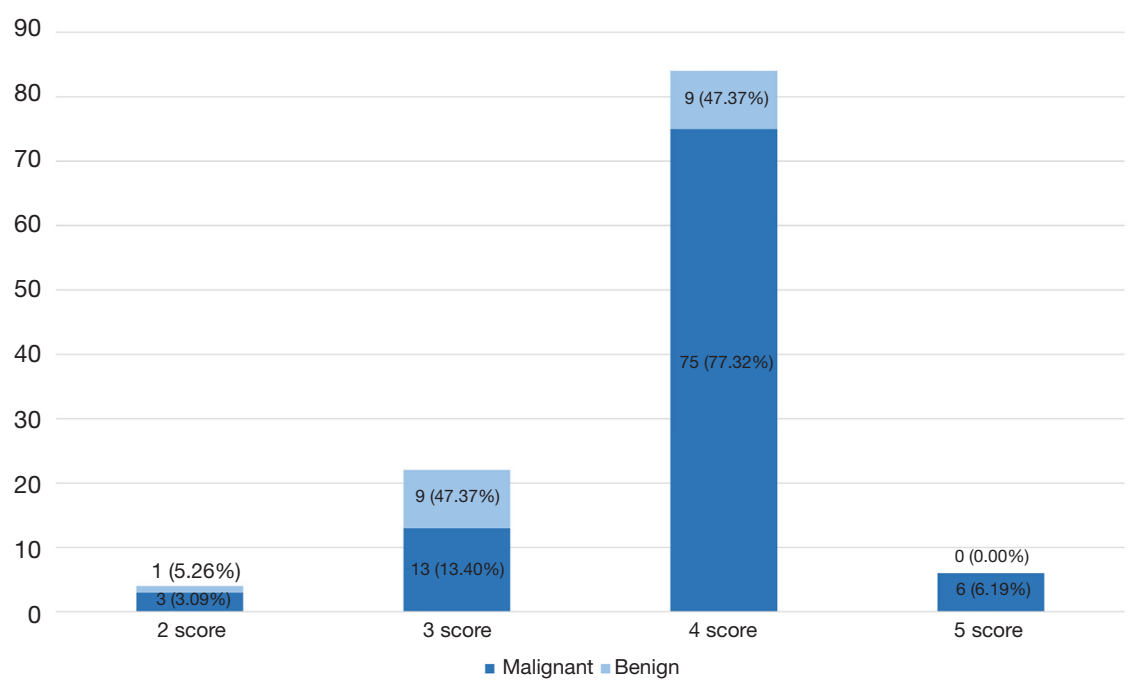

Figure 3 Distribution of diseases by elastography grading score. There were 3 cases of malignant lesions in score 2 , including 1 neuroendocrine tumor not otherwise specified, 1 squamous cell carcinoma and 1 adenocarcinoma. The benign lesion was inflammation. The 13 malignant lesions in score 3 were 7 adenocarcinoma, 1 squamous cell carcinoma, 4 small cell carcinoma and 1 NSCLS-NOS. The 9 benign masses in score 4 contained 5 tuberculosis, 3 inflammation and 1 aspergillosis. The 6 malignant tumors in score 5 included 1 typical carcinoid tumor, 1 small cell carcinoma, 2 adenocarcinoma, 1 squamous cell carcinoma and 1 NSCLS-NOS. NSCLS-NOS, non-small cell lung cancer not otherwise specified.

echoic feature indicating malignancy with a high sensitivity but a low specificity (20). Air-bronchogram appeared in four of the eight cases of inflammation in our study, but no air-bronchogram was found in tuberculosis. In general, the proportion of inflammation was small, and the specificity of air-bronchogram was only $21.05 \%$, without statistical difference between benign and malignant lung lesions.

The blood flow volume classification in this study came from breast cancer. However, we found that inflammatory masses and tuberculosis were also rich in blood flow; hence there was no significant statistical difference between malignant and benign lesions. This classification could not quantify blood flow, and vascular index and scattering index may be valuable for analyzing blood flow volume (32). Blood flow distribution in intrathoracic LNs has the value of predicting malignancy and hilar blood flow is a predictor of benign LNs (17). However, due to the absence of hilar structure in lung lesions, blood flow distribution was not analyzed in this study.

The optimal cutoff value of the elastography grading score was 3.5 , suggesting that the five-score method used for intrathoracic LNs previously was also applicable to lung lesions because a score $1-3$ was defined as benign (16). This elastography grading method was similar to color VAS score studied by Verhoeven et al. (33). Sun et al. found that the sensitivity and specificity of the elastography grading score for malignant LNs prediction were $85.71 \%$ and $81.82 \%$, respectively (16). The sensitivity in this study was $83.51 \%$, meaning a better predictive value for malignancy than benign. However, the specificity was only $52.63 \%$ in lung lesions, indicating that benign lesions in our study were relatively stiff, while elastography could only reflect the stiffness of lung lesions rather than the benign or malignant nature of lesions. Studies found that a history of pulmonary tuberculosis and apparent inhalation exposure to mineral dust such as coal or asbestos could influence the relative stiffness of LNs (34-36). Fibrosis in sarcoidosis granulomas may lead to false positive results of elastography grading score, similar to granulomatous fibrosis in tuberculosis $(28,37)$. Compared to inflammation, tuberculosis is stiffer and the elastography score were $3.25 \pm 0.71$ and $3.50 \pm 0.53$, respectively. However, the diagnostic accuracy of $78.45 \%$ for differentiating benign from malignant central lung lesions in our study was higher than the study using the elastography qualitative four-score method which showed a diagnostic accuracy of $73.7 \%$ (8).

The scoring model in our study found that when two or more features which had significant statistical differences 
occurred, the diagnostic efficiency of malignancy prediction could reach the best. Score 0 had a specificity of $84.21 \%$, which means the lesion was more likely to be benign when none of the four features were present. The sensitivity of score 1 for malignancy prediction was $96.91 \%$, that is, when at least one of the four characteristics appeared, the probability of false negative results was low. The PPV of score 3 and 4 were $93.65 \%$ and $95.45 \%$, that is, when at least 3 or 4 characteristics appeared, the lesion examined had a high probability to be malignant. The advantages of various EBUS characteristics could be complementary to achieve the best diagnostic efficiency. The scoring model can help the bronchoscopists to diagnose the benign and malignant lesions in real time during the EBUS-TBNA operation. When the pathological results are negative, it can also assist in the diagnosis of benign and malignant lesions. But pathological biopsy is still needed because the scoring model cannot replace pathological results.

This study still has some limitations. Patients were sequentially enrolled in a single specialized hospital where benign lesions account for a finite proportion, which might lead to unstable diagnostic effect of the scoring model. Data from multi-center studies that increase the proportion of benign lesions may lead to more objective and broadly applicable results. Second, this was a retrospective study and some lung lesions undergoing EBUS-TBNA might be omitted in the process of video collection. In addition, this scoring model was only constructed retrospectively without prospectively validation. Thus, a multi-center prospective study to validate the scoring model is worthy of further study.

\section{Conclusions}

For pulmonary lesions examined by EBUS-TBNA, when at least two of the four features (distinct margin, necrosis, lobulation sign and elastography grading score 4-5) were present on CP-EBUS images, the diagnostic accuracy for pulmonary lesions can reach the best of $87.07 \%$. The diagnostic scoring model constructed based on EBUS grayscale and elastography may have the potential in differentiating benign from malignant pulmonary lesions, especially in malignancy prediction.

\section{Acknowledgments}

Funding: This work was supported by the National Natural Science Foundation of China (grant number 81870078),
Shanghai Municipal Health and Medical Talents Training Program (grant number 2018BR09), and Shanghai Municipal Education Commission-Gaofeng Clinical Medicine Grant Support (grant number 20181815).

\section{Footnote}

Provenance and Peer Review: This article was commissioned by the editorial office, Fournal of Thoracic Disease for the series "Advance in Bronchoscopy for Peripheral Pulmonary Diseases". The article has undergone external peer review.

Reporting Checklist: The authors have completed the STARD reporting checklist. Available at http://dx.doi.org/10.21037/ jtd-2020-abpd-005

Data Sharing Statement: Available at http://dx.doi. org/10.21037/jtd-2020-abpd-005

Conflicts of Interest: All authors have completed the ICMJE uniform disclosure form (available at http://dx.doi. org/10.21037/jtd-2020-abpd-005). The series "Advance in Bronchoscopy for Peripheral Pulmonary Diseases" was commissioned by the editorial office without any funding or sponsorship. JS served as the unpaid Guest Editor of the series. The authors have no other conflicts of interest to declare.

Ethical Statement: The authors are accountable for all aspects of the work in ensuring that questions related to the accuracy or integrity of any part of the work are appropriately investigated and resolved. The study was conducted in accordance with the Declaration of Helsinki (as revised in 2013). The study was approved by institutional ethics board of Shanghai Chest Hospital (NO.: KS1946) and individual consent for this retrospective analysis was waived.

Open Access Statement: This is an Open Access article distributed in accordance with the Creative Commons Attribution-NonCommercial-NoDerivs 4.0 International License (CC BY-NC-ND 4.0), which permits the noncommercial replication and distribution of the article with the strict proviso that no changes or edits are made and the original work is properly cited (including links to both the formal publication through the relevant DOI and the license). See: https://creativecommons.org/licenses/by-nc-nd/4.0/. 


\section{References}

1. Bray F, Ferlay J, Soerjomataram I, et al. Global cancer statistics 2018: GLOBOCAN estimates of incidence and mortality worldwide for 36 cancers in 185 countries. CA Cancer J Clin 2018;68:394-424.

2. Gould MK, Donington J, Lynch WR, et al. Evaluation of individuals with pulmonary nodules: when is it lung cancer? Diagnosis and management of lung cancer, 3rd ed: American College of Chest Physicians evidence-based clinical practice guidelines. Chest 2013;143:e93S-120S.

3. Beckh S, Bölcskei PL, Lessnau KD. Real-time chest ultrasonography: a comprehensive review for the pulmonologist. Chest 2002;122:1759-73.

4. Diacon AH, Theron J, Bolliger CT. Transthoracic ultrasound for the pulmonologist. Curr Opin Pulm Med 2005;11:307-12.

5. Yasufuku K, Nakajima T, Chiyo M, et al. Endobronchial ultrasonography: current status and future directions. J Thorac Oncol 2007;2:970-9.

6. Gompelmann D, Eberhardt R, Herth FJ. Endobronchial ultrasound. Endosc Ultrasound 2012;1:69-74.

7. Kurimoto N, Murayama M, Yoshioka S, et al. Analysis of the internal structure of peripheral pulmonary lesions using endobronchial ultrasonography. Chest 2002;122:1887-94.

8. He HY, Chen JL, Ma H, et al. Value of Endobronchial Ultrasound Elastography in Diagnosis of Central Lung Lesions. Med Sci Monit 2017;23:3269-75.

9. Yasuda K, Mukai H, Nakajima M, et al. Staging of pancreatic carcinoma by endoscopic ultrasonography. Endoscopy 1993;25:151-5.

10. Rösch T, Braig C, Gain T, et al. Staging of pancreatic and ampullary carcinoma by endoscopic ultrasonography. Comparison with conventional sonography, computed tomography, and angiography. Gastroenterology 1992;102:188-99.

11. Zhi X, Chen J, Xie F, et al. Diagnostic value of endobronchial ultrasound image features: A specialized review. Endosc Ultrasound 2020. [Epub ahead of print]. doi: 10.4103/eus.eus_43_20.

12. Wahidi MM, Herth F, Yasufuku K, et al. Technical Aspects of Endobronchial Ultrasound-Guided Transbronchial Needle Aspiration: CHEST Guideline and Expert Panel Report. Chest 2016;149:816-35.

13. Zaleska-Dorobisz U, Kaczorowski K, Pawluś A, et al. Ultrasound elastography - review of techniques and its clinical applications. Adv Clin Exp Med 2014;23:645-55.

14. Sigrist RMS, Liau J, Kaffas AE, et al. Ultrasound
Elastography: Review of Techniques and Clinical Applications. Theranostics 2017;7:1303-29.

15. Dietrich CF, Jenssen C, Herth FJ. Endobronchial ultrasound elastography. Endosc Ultrasound 2016;5:233-8.

16. Sun J, Zheng X, Mao X, et al. Endobronchial Ultrasound Elastography for Evaluation of Intrathoracic Lymph Nodes: A Pilot Study. Respiration 2017;93:327-38.

17. Wang $\mathrm{L}, \mathrm{Wu} \mathrm{W}, \mathrm{Hu} \mathrm{Y}$, et al. Sonographic Features of Endobronchial Ultrasonography Predict Intrathoracic Lymph Node Metastasis in Lung Cancer Patients. Ann Thorac Surg 2015;100:1203-9.

18. Ren HY, Zhang XJ, Zhang K, et al. Endobronchial Ultrasound Combined with Clinical Data for Predicting Malignant Peripheral Pulmonary Lesions. Cancer Manag Res 2020;12:9837-44.

19. Parlamento S, Copetti R, Di Bartolomeo S. Evaluation of lung ultrasound for the diagnosis of pneumonia in the ED. Am J Emerg Med 2009;27:379-84.

20. Kuo CH, Lin SM, Chen HC, et al. Diagnosis of peripheral lung cancer with three echoic features via endobronchial ultrasound. Chest 2007;132:922-9.

21. Alici IO, Yilmaz Demirci N, Yilmaz A, et al. The sonographic features of malignant mediastinal lymph nodes and a proposal for an algorithmic approach for sampling during endobronchial ultrasound. Clin Respir J 2016;10:606-13.

22. Li F, Sone $\mathrm{S}$, Abe H, et al. Malignant versus benign nodules at CT screening for lung cancer: comparison of thin-section CT findings. Radiology 2004;233:793-8.

23. Hsu JS, Huang MS, Chen CY, et al. Correlation between EGFR mutation status and computed tomography features in patients with advanced pulmonary adenocarcinoma. J Thorac Imaging 2014;29:357-63.

24. Adler DD, Carson PL, Rubin JM, et al. Doppler ultrasound color flow imaging in the study of breast cancer: preliminary findings. Ultrasound Med Biol 1990;16:553-9.

25. Landis JR, Koch GG. The measurement of observer agreement for categorical data. Biometrics 1977;33:159-74.

26. Youden WJ. Index for rating diagnostic tests. Cancer 1950;3:32-5.

27. Kundel HL, Polansky M. Measurement of observer agreement. Radiology 2003;228:303-8.

28. Nachiappan AC, Rahbar K, Shi X, et al. Pulmonary Tuberculosis: Role of Radiology in Diagnosis and Management. Radiographics 2017;37:52-72.

29. Fujiwara T, Yasufuku K, Nakajima T, et al. The utility of sonographic features during endobronchial ultrasound- 
guided transbronchial needle aspiration for lymph node staging in patients with lung cancer: a standard endobronchial ultrasound image classification system. Chest 2010;138:641-7.

30. Gogia P, Insaf TZ, McNulty W, et al. Endobronchial ultrasound: morphological predictors of benign disease. ERJ Open Res 2016;2:00053-2015.

31. Reissig A, Kroegel C. Sonographic diagnosis and followup of pneumonia: a prospective study. Respiration 2007;74:537-47.

32. Kagawa T, Yuasa K, Fukunari F, et al. Quantitative evaluation of vascularity within cervical lymph nodes using Doppler ultrasound in patients with oral cancer: relation to lymph node size. Dentomaxillofac Radiol 2011;40:415-21.

33. Verhoeven RLJ, de Korte CL, van der Heijden E. Optimal Endobronchial Ultrasound Strain Elastography Assessment Strategy: An Explorative Study. Respiration

Cite this article as: Zhi X, Wang L, Chen J, Zheng X, Li Y, Sun J. Scoring model of convex probe endobronchial ultrasound multimodal imaging in differentiating benign and malignant lung lesions. J Thorac Dis 2020;12(12):7645-7655. doi: 10.21037/ jtd-2020-abpd-005
2019;97:337-47.

34. Uchimura K, Yamasaki K, Sasada S, et al. Quantitative analysis of endobronchial ultrasound elastography in computed tomography-negative mediastinal and hilar lymph nodes. Thorac Cancer 2020;11:2590-9.

35. Abedini A, Razavi F, Farahani M, et al. The utility of elastography during EBUS-TBNA in a population with a high prevalence of anthracosis. Clin Respir J 2020;14:488-94.

36. Uchimura K, Yamasaki K, Kawanami T, et al. Influential Factors for Assessing Endobronchial Ultrasound Elastography. Respiration 2020. [Epub ahead of print]. doi: 10.1159/000510643.

37. Livi V, Cancellieri A, Pirina P, et al. Endobronchial Ultrasound Elastography Helps Identify Fibrotic Lymph Nodes in Sarcoidosis. Am J Respir Crit Care Med 2019;199:e24-5. 